\title{
CUX2 functions as an oncogene in papillary thyroid cancer
}

This article was published in the following Dove Medical Press journal: OncoTargets and Therapy

\section{Yihan Sun* \\ Danrong Ye* \\ Yuefeng Li \\ Endong Chen \\ Rutian Hao \\ Yefeng Cai \\ Qingxuan Wang \\ Ouchen Wang \\ Xiaohua Zhang}

Department of Thyroid and Breast Surgery, The First Affiliated Hospital of Wenzhou Medical University,

Wenzhou 32500, China

*These authors contributed equally to this work
Correspondence: Xiaohua Zhang Department of Thyroid and Breast Surgery, The First Affiliated Hospital of Wenzhou Medical University, Wenzhou 32500, China

Email zhangxiaohualI26@163.com
Background: In recent years, the incidence of thyroid cancer (TC), the most common endocrine malignancy, has been increasing. Emerging evidence indicates that the CUT/CUX/CDP family of proteins can play an important role in tumor development and progression by regulating many cancer-related functions. However, the molecular functions of $C U X 2$ in TC remain unknown. Methods: In this study, we used a series of loss-of-function experiments and Western blot analysis to investigate the function of $C U X 2$ in TC and the mechanisms involved.

Results: Our data revealed that $C U X 2$ expression levels were upregulated in papillary thyroid cancer (PTC). Functionally, CUX2 silencing significantly inhibited PTC cell line (KTC-1 and BCPAP) proliferation, colony formation, migration, invasion, and apoptosis. Furthermore, CUX2 induced epithelial-mesenchymal transition (EMT) and influenced the phosphorylation of AKT and mTOR in the PI3K-AKT-mTOR pathways.

Conclusion: In summary, CUX2 may function as a tumor promoter in TC.

Keywords: papillary thyroid carcinoma, $C U X 2$, oncogene

\section{Introduction}

Thyroid cancer (TC) is the most frequent endocrine malignancy, with 53,990 estimated diagnosed cases and 2,060 estimated deaths in the United States in 2018. In recent years, its incidence has shown a significant upward trend worldwide. However, with the changes in clinical practice guidelines, TC incidence may appear to be decreasing, particularly among Whites. ${ }^{1,2}$ Papillary thyroid cancer (PTC) accounts for $80 \%-85 \%$ of all TCs. ${ }^{3}$ RAS mutations represent the second most identified genetic alteration in TC, and RAS-mutated PTC appears to be more aggressive and is associated with poor prognosis compared with other types of cancers. ${ }^{4,5}$ The PI3K/AKT pathway plays an extensive role in thyroid tumorigenesis, and its inhibitor can be a therapeutic target in TC. ${ }^{6}$

The CUT/CUX/CDP family of nuclear proteins was first reported in Drosophila; it plays an important role in mediating the dendrite branching pattern. ${ }^{7,8}$ Since then, a human version of CUT, named CUT-like homeobox 1 and 2 (CUX1 and CUX2), was subsequently identified as the mammalian orthologue of the Drosophila CUT gene. The full-length CUX proteins are characterized by four conserved DNA-binding domains, including the CUT homeodomain and three CUT repeat DNA-binding sequences (CR1, CR2, and CR3), each composed of 60-80 highly similar amino acids. ${ }^{9-11}$ Depending on the different cellular contexts, $C U X$ genes can express various isoforms and exhibit regulated expression levels, which might contribute to functional diversity. ${ }^{12,13} C U X$ genes are known to be associated with the initiation and progression of multiple diseases, such as brain diseases involved in synapse, dendrite, and axon development and various cancers. ${ }^{13-16}$ Paradoxically, $C U X$ has been implicated in cancer, both as a tumor 
suppressor and an oncogene, depending on distinct protein isoforms and perhaps on the dosage of gene expression. $C U X 1$ expression is upregulated in many advanced cancers, such as glioblastomas, colon rectal cancer, and breast cancer. ${ }^{17-19}$ However, some genetic and functional evidence also point that many cancers (uterine leiomyomas, breast cancer, acute myeloid leukemias, and myelo proliferative diseases) commonly exhibit loss or inactivation of one CUX1 allele, resulting in decreased expression and activity and promoting tumorigenesis. ${ }^{20-24}$ Increasing evidence implicate the notion that $C U X 1$, a haploinsufficient tumor suppressor gene, might be associated with tumor initiation, whereas increased copy number and expression promote tumor progression.

Unlike $C U X 1$, which is broadly expressed in many tissues, $C U X 2$ shows a more restricted expression pattern and is primarily expressed in the nervous system. ${ }^{25,26}$ Recently, Klampfl et $\mathrm{al}^{27}$ found that a mutation in $C U X 2$ is also linked with myeloproliferative neoplasms. $C U X 1$ and $C U X 2$ have a $48 \%$ amino acid identity. $C U X 2$ has several reported transcript variants. ${ }^{11}$ It exhibits similar DNA-binding specificities and binds to the same sequences as $C U X 1$, although its kinetics appear to be much more transient and rapid. ${ }^{28}$ CUX2 proteins have been found to bind not only to CCAATcontaining sites but also to other promoter sequences, acting as a repressor or an activator to regulate transcriptional activity in different contexts. ${ }^{29,30}$ Interestingly, in the liver, $C U X 2$ functions as a female-specific transcription activator and inhibits male-biased genes. ${ }^{31,32}$ In addition, $C U X 2$ is involved in various biological processes, including accelerating the repair of oxidative DNA damage, cell cycle progression, apoptotic signals, and other pathways. ${ }^{30,33-35}$

To date, few studies have focused on the relationship between $C U X 2$ and TC. Hence, in this study, we investigated the role of the $C U X 2$ gene in TC.

\section{Patients and methods}

\section{Patients and samples}

We selected 20 paired PTC tissues and matched noncancerous thyroid tissues from patients who underwent thyroid resection at the First Affiliated Hospital of Wenzhou Medical University. These tissues were flash frozen in liquid nitrogen immediately after surgery and stored at $-80^{\circ} \mathrm{C}$ before RNA isolation and quantitative real-time PCR (qRT-PCR) analysis. Further clinicopathological data were available. The use of all tissue samples in this study was approved by the ethics committee of the First Affiliated Hospital of Wenzhou Medical University, and written informed consent was obtained from each patient.

\section{Cell culture}

Human TC cell lines, KCT-1, TPC-1, BCPAP, FTC-133, and Htori-3, were provided by the Stem Cell Bank, Chinese Academy of Sciences. These cells were cultivated in RPMI 1640 (Thermo Fisher Scientific, Waltham, MA, USA) supplemented with 10\% FBS (Thermo Fisher Scientific), $1 \times$ Minimum Essential Medium nonessential amino acids (Thermo Fisher Scientific), and $1 \times$ sodium pyruvate (Thermo Fisher Scientific) and incubated in a humidified atmosphere containing $5 \% \mathrm{CO}_{2}$ at $37^{\circ} \mathrm{C}$.

\section{RNA extraction and qRT-PCR}

The total RNA was isolated using TRIzol reagent (Thermo Fisher Scientific) according to the manufacturer's instructions and then reverse transcribed into cDNA using a kit from Toyobo (Tokyo, Japan). qRT-PCR was performed in triplicate by using the THUNDERBIRD SYBR qPCR Mix (Toyobo) according to the manufacturer's instructions. The following gene-specific primers were used: CUX2 (F: 5'-TGAACCATAGGCACAACC-3'; R: 5'-AAACACCA AGAGGGGAAG-3') and GAPDH (F: 5'-GGTCGGAGTC AACGGATTTG-3'; R: 5'-ATGAGCCCCAGCCTT CTCCAT-3').

\section{RNA interference}

For knockdown studies, siRNA for $C U X 2$ was purchased from Shanghai Gene Pharma (Shanghai, China). Cell transfection was performed using RNAiMAX (Thermo Fisher Scientific) according to the manufacturer's protocol. The knockdown efficiency was confirmed by qRT-PCR and Western blot analyses.

\section{Cell proliferation and colony formation assays}

We utilized the colony formation and Cell Counting Kit-8 (CCK-8; Sigma-Aldrich Co., St Louis, MO, USA) assays to determine the proliferative ability. For the colony formation assay, the transfected KTC- 1 and BCPAP cells $\left(1.5 \times 10^{3} /\right.$ well $)$ were seeded in six-well plates. After 7 days, the cells were fixated with 4\% paraformaldehyde (PFA) for 30 minutes and stained with $0.1 \%$ crystal violet for 30 minutes. The colonies were counted only if they included at least 50 cells. For the proliferation assay, the transfected cells $\left(1.5 \times 10^{3}\right)$ were plated in 96-well plates and measured every 24 hours using the CCK-8 reagent following the manufacturer's instruction. The absorption was measured at $450 \mathrm{~nm}$ after adding the reagent and incubating for 2 hours in a $37^{\circ} \mathrm{C}$ incubator. All experiments were performed in triplicate. 


\section{Cell migration and invasion ability analyses}

Cellular migration and invasion assays were performed in a Boyden chamber system with a pore size of $8 \mathrm{~mm}$. For invasion assays, the inserts were coated with Matrigel matrix before cell seeding. The transfected cells $\left(4 \times 10^{5}\right.$ cells for KTC- 1 and $5 \times 10^{5}$ cells for BCPAP, double amount for invasion) were seeded in the upper chamber, and the chamber was placed into a 24 -well plate filled with a medium containing $20 \%$ FBS. The cells were incubated for 24 (KTC-1 cells) or 26 hours (BCPAP cells) at $37^{\circ} \mathrm{C}$. Then, the cells adhering to the lower surface of the membrane were fixed with $4 \%$ PFA for 30 minutes, stained with $0.01 \%$ crystal violet for 30 minutes, and photographed using a light microscope. All experiments were performed at least three times.

\section{Cell apoptosis assay}

Two days after infection, the cells were harvested and double stained with Annexin V conjugated to phycoerythrin and 7-aminoactinomycin (7-AAD) (Apoptosis Detection Kit-1; BD Pharmingen, San Diego, CA, USA). Apoptotic events were analyzed using FlowJo software. All these experiments were repeated in triplicate.

\section{Protein extraction and Western blot analysis}

The total cellular protein was extracted using a RIPA protein lysis buffer (Beyotime, Shanghai, China). Equal amounts of protein $(20 \mu \mathrm{g})$ were loaded and separated by SDS-PAGE and transferred onto the polyvinylidene fluoride membrane. After blocking with 5\% skimmed milk, the membrane was incubated with a relative antibody (Abcam, Cambridge, UK) overnight at $4^{\circ} \mathrm{C}$. Then, the membrane was washed and incubated with horseradish peroxidase-linked secondary anti-goat immunoglobulin $\mathrm{G}$ antibody (Abcam) at room temperature for 1 hour. GAPDH was used as internal control. All experiments were performed at least three times.

\section{Statistical analyses}

The data are expressed as mean $\pm \mathrm{SD}$. The different gene expression levels of $C U X 2$ in tumor tissues were analyzed using paired sample $t$-test. We used the $t$-test or one-way ANOVA test for continuous variables. All $P$-values were two sided, and a $P$-value of $<0.05$ was considered statistically significant. Statistical analysis was performed with SPSS software version 19.0 (IBM Corporation, Armonk, NY, USA). GraphPad Prism 5 (GraphPad Software, Inc., La Jolla, CA, USA) was used for the graphs.

\section{Results CUX2 is overexpressed in TC}

To evaluate the expression level of $C U X 2$ in PTC, qRTPCR analysis was performed on 20 paired PTC tissues and adjacent noncancerous thyroid tissues. Results showed that $C U X 2$ was significantly higher than the corresponding normal tissues $(P<0.05$; Figure $1 \mathrm{~A})$. Meanwhile, we also assessed the mRNA expression levels of CUX2 in TC cell lines and found that $C U X 2$ was consistently upregulated in KCT-1, TPC-1, BCPAP, and FTC-133 cells compared with the normal thyroid cell line, Htori-3 (Figure 1B).

\section{CUX2 knockdown decreases proliferation and colony formation}

To investigate the functional role of $C U X 2$ in papillary thyroid cell lines, we sequentially performed loss-of-function experiments to explore the biological effect of $C U X 2$. Considering that the TPC-1 cell viability worsened after siRNA treatment, the KTC- 1 and BCPAP cells were finally selected to be transfected with siRNA targeting $C U X 2$. The results of qRT-PCR and Western blot analyses showed that the expression of $C U X 2$ was successfully decreased $(>30 \%)$ both at the mRNA and protein levels (Figures $1 \mathrm{C}$ and $3 \mathrm{C}$ ). Then, we performed colony formation and CCK-8 assays to investigate the biological role of $C U X 2$. The colony formation assay indicated that $C U X 2$ knockdown inhibited the proliferation in KTC-1 and BCPAP cells compared with the control group (Figure 2A and D). Furthermore, the CCK-8 assay (Figure $2 \mathrm{G}$ and $\mathrm{H}$ ) showed a consistent result with the colony formation assay. The proliferative capacity of the cell lines transfected with siRNA-CUX2 was significantly attenuated. These data indicated that downregulation of CUX2 can suppress the proliferation and growth abilities of KTC-1 and BCPAP cells.

\section{CUX2 knockdown impairs the migration and invasion}

Given that cell migration and invasion are critical steps for cancer metastasis, we carried out migration and invasion assays to further identify whether the knockdown of CUX2 expression can regulate TC metastasis abilities. We performed cell migration assay using the Transwell chamber migration assay. The cancer cells with $C U X 2$ knockdown displayed fewer cells that migrated through the membrane after 24 hours than cells transfected with the negative control. We then examined the number of cells that penetrated through the Matrigel in a Transwell chamber to investigate the invasiveness. Consistently, the invasion assays showed the same tendency, 

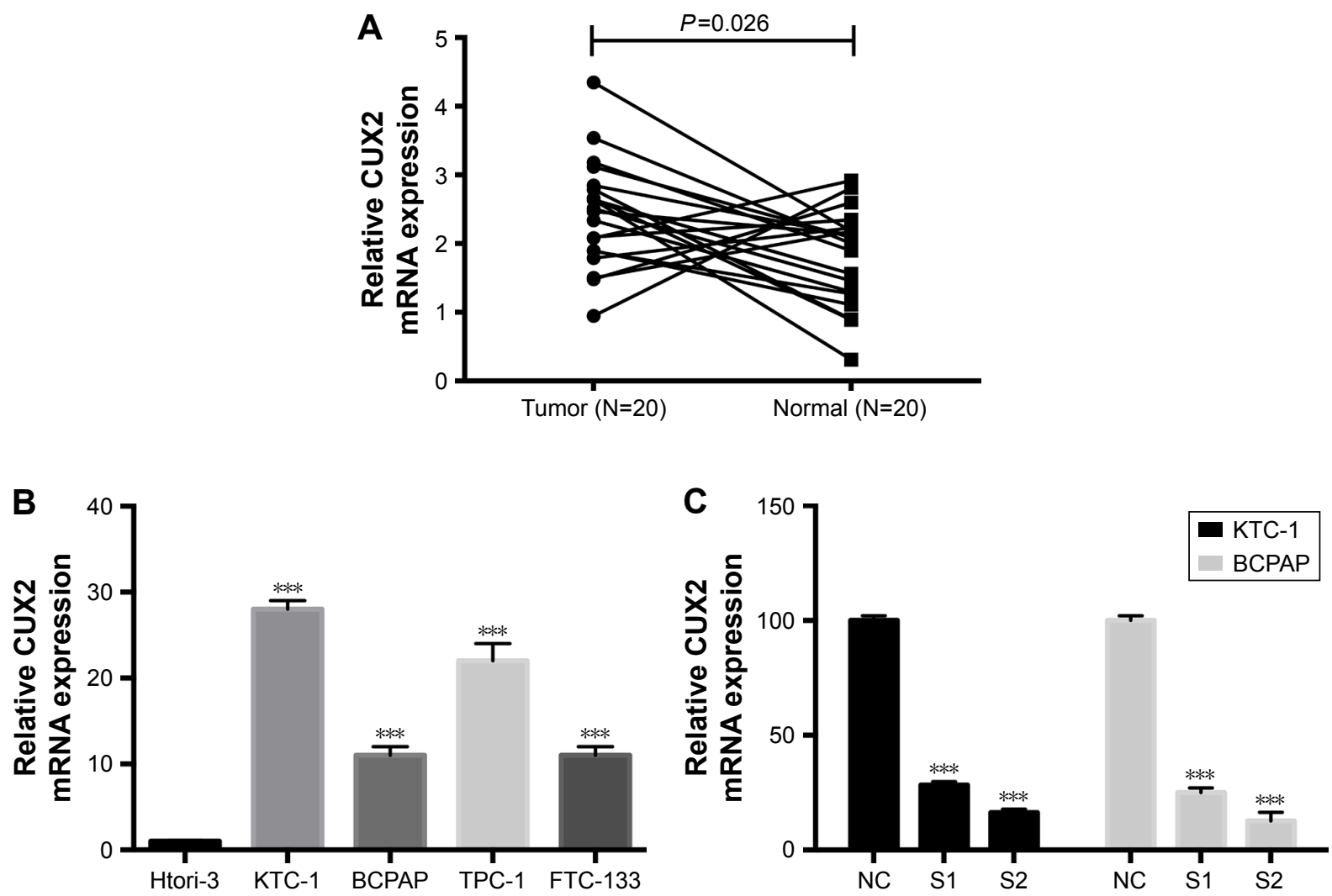

Figure I CUX2 gene is overexpressed in thyroid cancer and knockdown CUX2 gene in thyroid cancer cell lines.

Notes: (A) mRNA expression of CUX2 in our clinical PTC tissues $(n=20)$. (B) mRNA expression levels of CUX2 in TC cell lines and a control cell line. (C) qRT-PCR analysis of the knockdown efficiency of CUX2 in KTC-I and BCPAP cells treated with two specific siRNAs. Data represent mean \pm SD from three independent experiments. $* * * P<0.001$ compared with siRNA-CUX2 and siRNA-negative control.

Abbreviations: qRT-PCR, quantitative real-time PCR; PTC, papillary thyroid cancer; TC, thyroid cancer.

that is, CUX2 knockdown dramatically attenuated the invasion capacity compared with vector-transfected cells (Figure 2B, C, E, and F). Therefore, CUX2 knockdown has a significant role in inhibiting tumor metastasis in TC cell lines.

\section{CUX2 knockdown induces apoptosis}

To further explore the possible mechanism of the abovementioned observations, we used 7-AAD and Annexin V staining in KTC-1 and BCPAP cells after different treatments to analyze cell death and apoptosis by flow cytometry. We found that the percentage of apoptotic cells markedly increased in both cell lines compared with that of the control group (Figure 3A and $\mathrm{B}$ ). These findings suggested that CUX2 knockdown caused proliferation, and metastasis arrest might trigger cell apoptosis.

\section{CUX2 facilitates cell migration and invasion by regulating the expression of E-cadherin and vimentin}

Emerging evidence has verified that the epithelialmesenchymal transition (EMT) is an integral process that involves cancer invasion, metastasis, and other tumor progression behaviors. ${ }^{36,37}$ Meanwhile, the deregulation or loss of the expression of E-cadherin plays a fundamental role in the EMT process. Inversely, upregulation of the expression of vimentin, a mesenchymal associated marker, contributes to cell metastasis. ${ }^{38-40}$ On the basis of our previous observation that $C U X 2$ promotes PTC cell invasion, we next investigated whether $C U X 2$ can mediate the EMT process of PTC cell lines. Through Western blot analysis, we observed that the expression levels of E-cadherin were increased, whereas those of vimentin were obviously downregulated in CUX2 knockdown cells compared with the vector-transfected cells (Figure 3C). These results indicate that the expression of $C U X 2$ might regulate the expression of E-cadherin and vimentin, thereby affecting the EMT process.

\section{PI3K-AKT-mTOR pathway is involved in the regulatory effects of CUX2}

The PI3K-AKT-mTOR pathway, an important survival pathway that is activated in many types of cancers, regulates cellular metabolism, tumor proliferation, and apoptosis. ${ }^{41}$ 

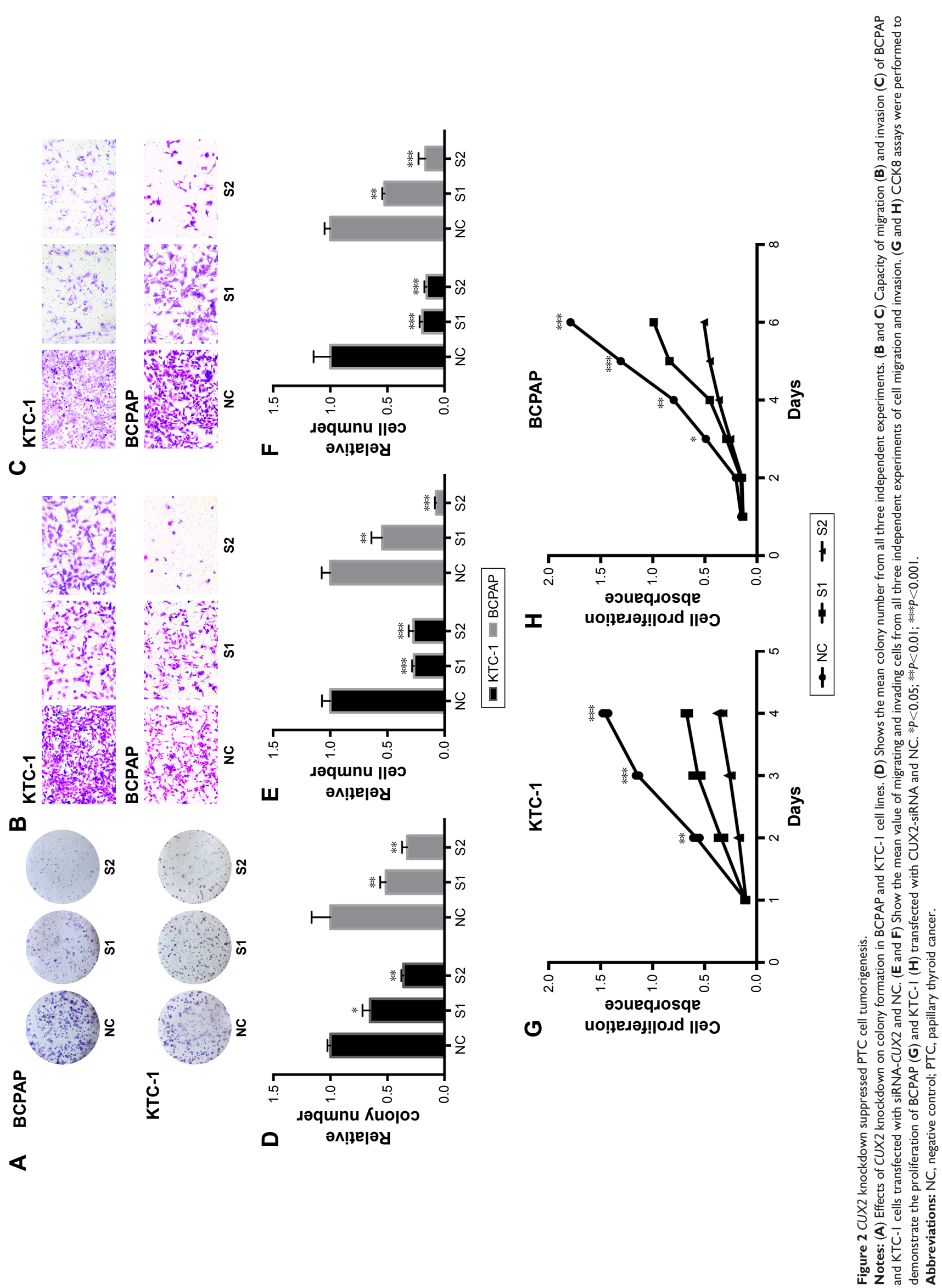
A
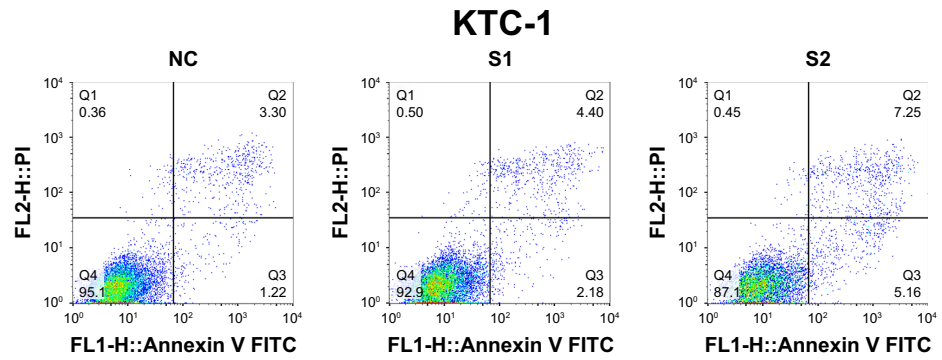

\section{C}

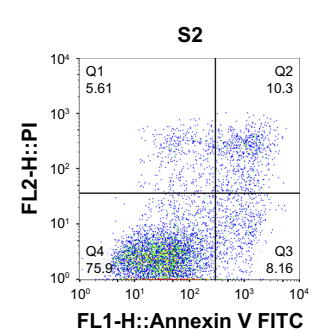

BCPAP
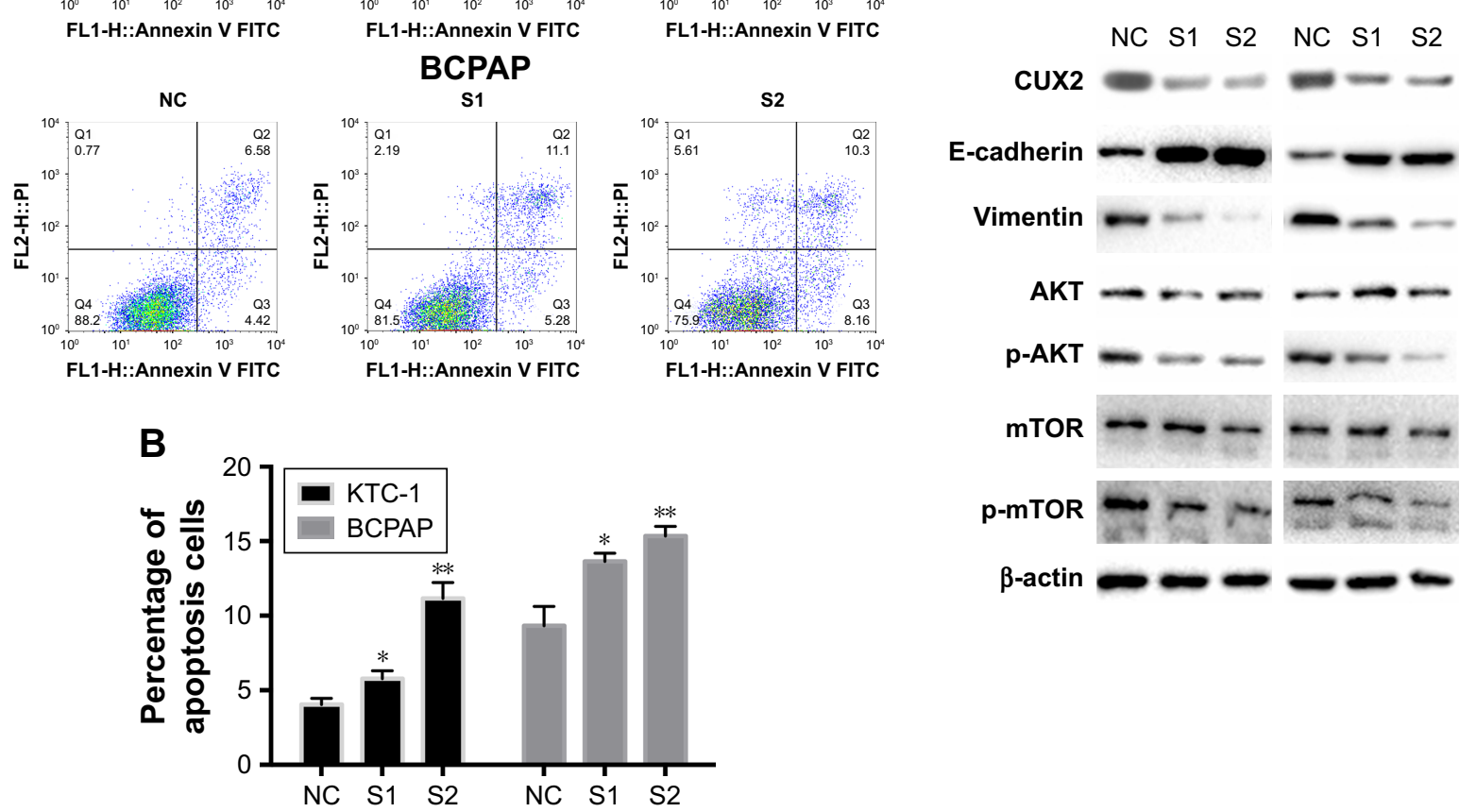

Figure 3 CUX2 knockdown induced PTC cell line apoptosis, and the EMT and PI3K-AKT-mTOR pathways were involved in CUX2's effect.

Notes: (A) Effects of CUX2 knockdown on cell apoptosis. Cells transfected with siRNA had more apoptosis cells as determined by Annexin V/PI staining and flow cytometry analysis. (B) Data represent mean of the percentage of apoptosis cells from all three independent experiments. (C) PTC cells were transfected with siRNA-CUX2 and NC. The expression and phosphorylation of key molecules of the pathways were assessed via Western blot analysis. $\beta$-Actin was used as an internal control. $* P<0.05 ; * * P<0.01$. Abbreviations: EMT, epithelial-mesenchymal transition; NC, negative control; PI, propidium iodide; PTC, papillary thyroid cancer.

As critical downstream effectors, AKT and mTOR are frequently hyperactivated in human cancers. ${ }^{42}$ To determine whether the PI3K-AKT-mTOR pathway is a potential cancerrelated pathway of $C U X 2$ in TC cells, we further investigated the expression of relevant proteins by Western blot analysis. As shown in Figure 3C, the phosphorylation of AKT and mTOR decreased in both PTC cell lines transfected with CUX2 siRNA, whereas total AKT and mTOR were not significantly altered. According to these results, we can speculate that the PI3K-AKT-mTOR pathway may be associated with the mechanism of CUX2 in promoting tumorigenesis in PTC.

\section{Discussion}

In this study, our experimental evidence supports that the CUX2 gene may play an oncogenic role in PTC cell lines. The results can be summarized as follows. First, we demonstrated that $C U X 2$ exhibits higher expression levels in PTC tissues and cell lines compared with the control. Moreover, we also found that $C U X 2$ knockdown attenuated the ability of proliferation and inhibited the migration and invasion. The suppression of CUX2 can activate the apoptosis of PTC cell lines. Interestingly, this is reminiscent of the important role of $C U X 2$ in regulating proliferation and cell cycle length of the nervous system. ${ }^{30} \mathrm{Pal}$ et al previously found that $C U X 2$ knockdown reduces proliferation and increases apoptosis in breast cancer cells. ${ }^{35}$

In addition, the credible data strongly implied that $C U X 1$ and $C U X 2$ displayed a highly conserved sequence and thus exhibited very similar molecular functions. ${ }^{10,11}$ Previously, experimental evidence showed the role of $C U X 1$ in promoting cell migration and invasion in numerous tumor cells by regulating TGF- $\beta$ signaling and EMT. ${ }^{18,43}$ The EMT process has been proven to have a strong association with cancer invasion and migration. ${ }^{44}$ Meanwhile, our data revealed that CUX2 silencing results in E-cadherin upregulation and vimentin downregulation. Therefore, we presumed that $C U X 2$ may play an important role in PTC cell metastasis via reversing the EMT process. Furthermore, a previous report on CUXI 
as a downstream effector of the PI3K-AKT pathway can confer resistance to apoptosis in pancreatic cancer, ${ }^{45}$ lending credence to the possibility of CUX2 regulating TPC cells' function via the PI3K-AKT-mTOR pathway. Meanwhile, previous reports demonstrated that the PI3K-AKT-mTOR pathway is one of the cardinal pathways in most malignancies, including TC. ${ }^{46-48}$ The PI3K-AKT-mTOR pathways have been demonstrated to regulate cell proliferation, apoptosis, and differentiation. We found that when CUX2 expression levels changed, phosphorylated AKT and mTOR were altered compared with total AKT and mTOR. Altogether, these results suggest that $C U X 2$ may affect the protein phosphorylation of the PI3K-AKT-mTOR pathway and promote PTC tumorigenesis.

Moreover, the present observations provide evidence that CUX2 may function as a tumor-promotive gene in regulating PTC cell proliferation and progression. However, this work has some limitations. First, we analyzed the association between $C U X 2$ expression and clinicopathological features in our clinical samples but failed to find a statistically significant relationship. This is probably due to the small number of patients. Second, whether $C U X 2$ expression can influence the tumorigenesis of PTC cells in vivo should be further studied. In addition, previous studies have verified that $C U X$ gene knockdown impairs the function of repairing oxidative DNA. In RAS-driven tumor cells, which tend to generate higher levels of ROS, CUXI knockdown could exhibit synthetic lethal effect in cancer cells. ${ }^{35,49}$ In this regard, exploring the relationship between $C U X 2$ and RAS mutation in TC may be interesting.

\section{Conclusion}

CUX2 may act as a tumor promoter gene in PTC via regulating EMT and influencing the phosphorylation of AKT and mTOR in the PI3K-AKT-mTOR pathways.

\section{Acknowledgment}

This work was supported by grants from the Major Science and Technology Projects of Zhejiang Province (2015C03052).

\section{Disclosure}

The authors report no conflicts of interest in this work.

\section{References}

1. La Vecchia C, Malvezzi M, Bosetti C, et al. Thyroid cancer mortality and incidence: a global overview. Int J Cancer. 2015;136(9):2187-2195.

2. Siegel RL, Miller KD, Jemal A, Ajd P. Cancer statistics, 2018. CA Cancer J Clin. 2018;68(1):7-30.

3. Fahiminiya S, de Kock L, Foulkes WD. Biologic and clinical perspectives on thyroid cancer. New Engl J Med. 2011;375(11):1054.
4. Garcia-Rostan G, Zhao H, Camp RL, et al. Ras mutations are associated with aggressive tumor phenotypes and poor prognosis in thyroid cancer. J Clin Oncol. 2003;21(17):3226-3235.

5. Howell GM, Hodak SP, Yip L. RAS mutations in thyroid cancer. Oncologist. 2013;18(8):926-932.

6. Hou P, Liu D, Shan Y, et al. Genetic alterations and their relationship in the phosphatidylinositol 3-kinase/Akt pathway in thyroid cancer. Clin Cancer Res. 2007;13(4):1161-1170.

7. Blochlinger K, Bodmer R, Jan LY, Jan YN. Patterns of expression of cut, a protein required for external sensory organ development in wild-type and cut mutant Drosophila embryos. Genes Dev. 1990;4(8):1322-1331.

8. Grueber WB, Jan LY, Jan YN. Different levels of the homeodomain protein cut regulate distinct dendrite branching patterns of Drosophila multidendritic neurons. Cell. 2003;112(6):805-818.

9. Blochlinger K, Bodmer R, Jack J, Jan LY, Jan YN. Primary structure and expression of a product from cut, a locus involved in specifying sensory organ identity in Drosophila. Nature. 1988;333(6174):629-635.

10. Neufeld EJ, Skalnik DG, Lievens PM, Orkin SH. Human CCAAT displacement protein is homologous to the Drosophila homeoprotein, cut. Nat Genet. 1992;1(1):50-55.

11. Quaggin SE, Heuvel GB, Golden K, Bodmer R, Igarashi P. Primary structure, neural-specific expression, and chromosomal localization of Cux-2, a second murine homeobox gene related to Drosophila cut. J Biol Chem. 1996;271(37):22624-22634.

12. Harada R, Vadnais C, Sansregret L, et al. Genome-wide location analysis and expression studies reveal a role for p110 CUX1 in the activation of DNA replication genes. Nucleic Acids Res. 2008;36(1):189-202.

13. Ferrere A, Vitalis T, Gingras H, Gaspar P, Cases O. Expression of Cux-1 and Cux-2 in the developing somatosensory cortex of normal and barrel-defective mice. Anat Rec A Discov Mol Cell Evol Biol. 2006; 288(2):158-165.

14. Sarrazin S, d'Albis MA, McDonald C, et al. Corpus callosum area in patients with bipolar disorder with and without psychotic features: an international multicentre study. J Psychiatry Neurosci. 2015;40(5):352-359.

15. Sarrazin S, Poupon C, Linke J, et al. A multicenter tractography study of deep white matter tracts in bipolar I disorder: psychotic features and interhemispheric disconnectivity. JAMA Psychiatry. 2014;71(4):388-396.

16. Glaser B, Kirov G, Green E, Craddock N, Owen MJ. Linkage disequilibrium mapping of bipolar affective disorder at 12q23-q24 provides evidence for association at CUX2 and FLJ32356. Am J Med Genet B Neuropsychiatr Genet. 2005;132B(1):38-45.

17. Cancer Genome Atlas Network. Comprehensive molecular characterization of human colon and rectal cancer. Nature. 2012;487(7407): 330-337.

18. Michl P, Ramjaun AR, Pardo OE, et al. CUTL1 is a target of TGF(beta) signaling that enhances cancer cell motility and invasiveness. Cancer Cell. 2005;7(6):521-532.

19. Kaur S, Ramdzan ZM, Guiot MC. CUX1 Stimulates APE1 Enzymatic Activity and Increases the Resistance of Glioblastoma Cells to the Mono-Alkylating Agent, Temozolomide. Neuro Oncol. 2017;20(4): 484-493.

20. Wong CC, Martincorena I, Rust AG, et al. Inactivating CUX1 mutations promote tumorigenesis. Nat Genet. 2014;46(1):33-38.

21. Zeng WR, Watson P, Lin J, et al. Refined mapping of the region of loss of heterozygosity on the long arm of chromosome 7 in human breast cancer defines the location of a second tumor suppressor gene at 7q22 in the region of the CUTL1 gene. Oncogene. 1999;18(11):2015-2021.

22. Zeng WR, Scherer SW, Koutsilieris M, et al. Loss of heterozygosity and reduced expression of the CUTL1 gene in uterine leiomyomas. Oncogene. 1997;14(19):2355-2365.

23. Thoennissen NH, Lasho T, Thoennissen GB, Ogawa S, Tefferi A, Koeffler HP. Novel CUX1 missense mutation in association with 7q-at leukemic transformation of MPN. Am J Hematol. 2011;86(8):703-705.

24. Pedersen-Bjergaard J, Andersen MT, Andersen MK. Genetic pathways in the pathogenesis of therapy-related myelodysplasia and acute myeloid leukemia. Hematology Am Soc Hematol Educ Program. 2007; 2007(1):392-397. 
25. Iulianella A, vanden Heuvel G, Trainor P. Dynamic expression of murine Cux2 in craniofacial, limb, urogenital and neuronal primordia. Gene Expr Patterns. 2003;3(5):571-577.

26. Bachy I, Franck MC, Li L, Abdo H, Pattyn A, Ernfors P. The transcription factor Cux2 marks development of an A-delta sublineage of TrkA sensory neurons. Dev Biol. 2011;360(1):77-86.

27. Klampfl T, Harutyunyan A, Berg T, et al. Genome integrity of myeloproliferative neoplasms in chronic phase and during disease progression. Blood. 2011;118(1):167-176.

28. Gingras H, Cases O, Krasilnikova M, Bérubé G, Nepveu A. Biochemical characterization of the mammalian Cux2 protein. Gene. 2005;344: 273-285.

29. Cubelos B, Sebastián-Serrano A, Beccari L, et al. Cux1 and Cux2 regulate dendritic branching, spine morphology, and synapses of the upper layer neurons of the cortex. Neuron. 2010;66(4):523-535.

30. Cubelos B, Sebastián-Serrano A, Kim S, et al. Cux-2 controls the proliferation of neuronal intermediate precursors of the cortical subventricular zone. Cereb Cortex. 2008;18(8):1758-1770.

31. Conforto TL, Zhang Y, Sherman J, Waxman DJ. Impact of CUX2 on the female mouse liver transcriptome: activation of female-biased genes and repression of male-biased genes. Mol Cell Biol. 2012;32(22): 4611-4627.

32. Conforto TL, Steinhardt GF 4th, Waxman DJ. Cross Talk Between GH-Regulated Transcription Factors HNF6 and CUX2 in Adult Mouse Liver. Mol Endocrinol. 2015;29(9):1286-1302.

33. Iulianella A, Sharma M, Durnin M, Vanden Heuvel GB, Trainor PA. Cux2 (Cut12) integrates neural progenitor development with cell-cycle progression during spinal cord neurogenesis. Development. 2008; 135(4):729-741.

34. Wittmann W, Iulianella A, Gunhaga L. Cux2 acts as a critical regulator for neurogenesis in the olfactory epithelium of vertebrates. Dev Biol. 2014;388(1):35-47.

35. Pal R, Ramdzan ZM, Kaur S, et al. CUX2 protein functions as an accessory factor in the repair of oxidative DNA damage. J Biol Chem. 2015;290(37):22520-22531.

36. Nieto MA. Epithelial plasticity: a common theme in embryonic and cancer cells. Science. 2013;342(6159):1234850.
37. Yang J, Weinberg RA. Epithelial-mesenchymal transition: at the crossroads of development and tumor metastasis. Dev Cell. 2008;14(6):818-829.

38. Theys J, Jutten B, Habets R, et al. E-Cadherin loss associated with EMT promotes radioresistance in human tumor cells. Radiother Oncol. 2011; 99(3):392-397.

39. Zeisberg M, Neilson EG. Biomarkers for epithelial-mesenchymal transitions. J Clin Invest. 2009;119(6):1429-1437.

40. Lamouille S, Xu J, Derynck R. Molecular mechanisms of epithelialmesenchymal transition. Nat Rev Mol Cell Biol. 2014;15(3):178-196.

41. Lopiccolo J, Blumenthal GM, Bernstein WB, Dennis PA. Targeting the PI3K/Akt/mTOR pathway: effective combinations and clinical considerations. Drug Resist Updat. 2008;11(1-2):32-50.

42. Hay N. The Akt-mTOR tango and its relevance to cancer. Cancer Cell. 2005;8(3):179-183.

43. Malizia AP, Lacey N, Walls D, Egan JJ, Doran PP. CUX1/Wnt signaling regulates epithelial mesenchymal transition in EBV infected epithelial cells. Exp Cell Res. 2009;315(11):1819-1831.

44. Yilmaz M, Christofori G. EMT, the cytoskeleton, and cancer cell invasion. Cancer Metastasis Rev. 2009;28(1-2):15-33.

45. Ripka S, Neesse A, Riedel J, et al. CUX1: target of Akt signalling and mediator of resistance to apoptosis in pancreatic cancer. Gut. 2010; 59(8):1101-1110.

46. Liu D, Hou P, Liu Z, Wu G, Xing M. Genetic alterations in the phosphoinositide 3-kinase/Akt signaling pathway confer sensitivity of thyroid cancer cells to therapeutic targeting of Akt and mammalian target of rapamycin. Cancer Res. 2009;69(18):7311-7319.

47. Viglietto G, Amodio N, Malanga D, Scrima M, Mc D. Contribution of PKB/AKT signaling to thyroid cancer. Front Biosci (Landmark Ed). 2011;16:1461-1487.

48. Hou P, Ji M, Xing M. Association of PTEN gene methylation with genetic alterations in the phosphatidylinositol 3-kinase/AKT signaling pathway in thyroid tumors. Cancer. 2008;113(9):2440-2447.

49. Ramdzan ZM, Vadnais C, Pal R, et al. RAS transformation requires CUX1-dependent repair of oxidative DNA damage. PLoS Biol. 2014; 12(3):e1001807.
OncoTargets and Therapy

\section{Publish your work in this journal}

OncoTargets and Therapy is an international, peer-reviewed, open access journal focusing on the pathological basis of all cancers, potential targets for therapy and treatment protocols employed to improve the management of cancer patients. The journal also focuses on the impact of management programs and new therapeutic agents and protocols on

\section{Dovepress}

patient perspectives such as quality of life, adherence and satisfaction The manuscript management system is completely online and includes a very quick and fair peer-review system, which is all easy to use. Visit http://www.dovepress.com/testimonials.php to read real quotes from published authors. 